\title{
La educación superior en Chile y el rol del mercado: ¿culpable o inocente?
}

\author{
Higher education in Chile and the role of the market: guilty or innocent? \\ Emilio Rodríguez Ponce ${ }^{1}$ \\ Recibido 30 de agosto de 2011, aceptado 28 de marzo de 2012 \\ Received: August 30, 2011 Accepted: March 28, 2012
}

\begin{abstract}
RESUMEN
El siguiente artículo analiza las deficiencias del actual sistema de educación superior chileno y la crisis en la que se encuentra, señalando que el éxito de éste no puede medirse por los resultados económicos que puede generar, sino por la permanente labor interna de sus actores y su aporte a la sociedad. Primeramente, se analiza el sistema de licenciamiento de las instituciones privadas y los estándares de calidad poco exigentes que no han asegurado la excelencia y han permitido que algunas operen con evidentes fines de lucro. Posteriormente, se analiza el funcionamiento del sistema de financiamiento, sus paradojas y la falta de regulación. Finalmente, se plantean ineludibles cuestionamientos acerca de la libertad para elegir dónde estudiar, la generación de bienes valiosos para la sociedad y la suficiencia de los niveles de cobertura, calidad y equidad del modelo de educación superior. Con lo anterior, se concluye que el sistema presenta una cobertura creciente pero no muestra la problemática que surge en su interior, con altos niveles de deserción, duración excesiva de los estudios, falta de equidad en el acceso y la pérdida de credibilidad en la calidad institucional. Pero es imposible pensar que el mercado, por sí solo, sea el responsable de dar solución a las reflexiones planteadas sobre condición socioeconómica del estudiante, estandarización de los costos de la educación, movilidad de recursos en el sistema, costo de traslado del usuario y asimetría de la información al momento de tomar decisiones sobre educación superior.
\end{abstract}

Palabras clave: Sistema de licenciamiento, instituciones privadas, acreditación, estándares de excelencia, lucro, sistema de financiamiento, cobertura, calidad y equidad del modelo de educación superior chileno.

\section{ABSTRACT}

The following article analyzes the deficiencies of the current Chilean system of higher education and the crisis that it is, indicating that the success of this one can not measure up for the economic results that this one can generate, but by the permanent internal labor of his actors and his contribution to society. Firstly, it is analyzed the discharge of private institution along with the slightly demanding standards of quality of those have not assured the excellence and have allowed that someone operates with evident profit making. Subsequently, the financing system running, his paradoxes and the lack of regulation are analyzed. Finally, this raises unavoidable questions about the liberty of choosing where to study, the generation of valuable goods to society and the insufficiency of coverage levels, quality and equity of the higher education model. With the previous thing, it can conclude that the system shows an increasing coverage but does not show the situation that arises inside, with high levels of desertion, excessive duration of the studies, lack of equity in the access and the loss of credibility in the institutional quality. But it is impossible to think that the market by his own, is the responsible entity of give solution to the presented reflections about socioeconomic condition of the students, standardization of the costs of the education, of resources mobility in the system, cost of transfer of the user and asymmetry of the information at once to take decisions related with higher education.

Keywords: Discharge system, private institutions, accreditation, standards of excellence, profit, financing system, coverage, quality and equity of the Chilean model of higher education.

1 Universidad de Tarapacá. Arica, Chile. E-mail: erodrigu@uta.cl 


\section{INTRODUCCIÓN}

Aun siendo muy generosos en la evaluación del actual sistema de educación superior de Chile, se debe concluir que éste es deficiente, que está en crisis y que no responde a los requerimientos más esenciales de una sociedad en la era del conocimiento. En efecto, el sistema no responde ni a la lógica de un modelo que busca la competitividad del país, ni mucho menos a la lógica de un sistema que busca en la educación superior el mejoramiento cualitativo del hombre y de la sociedad.

El punto es que el éxito de un sistema o modelo de educación superior no se mide por los beneficios económicos que éste genera para quienes imparten la educación, o por las oportunidades de inversión que ofrece a empresas o grupos multinacionales, o a los fondos de inversión, a las inmobiliarias o a las agencias de publicidad que operan en él. No se mide tampoco por las rentas percibidas por los distintos actores o proveedores de la educación terciaria. El éxito y la calidad del sistema se asocia indisolublemente al quehacer de los miembros de las comunidades de educación superior internamente y en su interacción con la sociedad.

En 1981 se llevó a cabo una reforma de la educación superior en Chile [22], la que consistentemente con el accionar del gobierno de aquella época no dio lugar a discusión ni debate democrático de ningún tipo. Dicha reforma, simplemente, se diseñó y se impuso. Los posteriores gobiernos democráticos, que emergen a partir de 1990, paradójicamente avanzan, sin solución de continuidad, en la misma lógica impulsada por el gobierno militar. Ciertamente, el Poder Ejecutivo desde el retorno a la democracia sólo ha realizado ajustes menores al modelo de los años 80 .

Tales ajustes, lejos de debilitar el sistema y su diseño previo, lograron su consolidación en el mundo de las ideas, al menos entre los miembros del "establishment", y sobre todo en el campo de los hechos reales, en el que han debido vivir las comunidades que conforman la educación superior del país.

\section{MARCO TEÓRICO}

En este contexto, se debe destacar en primer lugar la generación de un sistema de licenciamiento
[22] para las instituciones privadas, con exigencias ajustadas a los propósitos institucionales, que convenientemente en muchos casos fueron propósitos modestos, a fin de cumplir con estándares, más bien, poco exigentes. Considérese, por ejemplo, que 36 instituciones universitarias superaron los procesos de licenciamiento, y paradójicamente algunas de ellas no fueron capaces de salvar con éxito los procesos iniciales de acreditación. Esto en atención, principalmente, a que sus propuestas de desarrollo y propósitos iniciales fueron cambiados substancialmente al obtener su licenciamiento. Fue así como hubo instituciones que generaron carreras de alta demanda, probablemente en muchos casos sin la debida consideración de criterios de calidad. A consecuencia de lo anterior, la geografía nacional vio nacer sedes de dudosa calidad de norte a sur.

El licenciamiento no garantizó, en absoluto, el cumplimiento de estándares de excelencia, entre otras cosas porque no era su finalidad. Al contrario, validó el sistema de instituciones privadas, dándoles una licencia de legalidad y legitimidad para operar. Así, se consolidó la concepción de un mercado para la educación superior, consiguiéndose para estas instituciones la aceptación del "establishment" y también de los potenciales estudiantes. Se licenciaron instituciones que, al menos en algunos casos, buscaban a través de sus vínculos con empresas relacionadas de los mismos fundadores el lucro para dichos propietarios.

Claramente, el sistema de licenciamiento, por la vía de los hechos concretos, dio sustento a que algunas de las universidades privadas trabajasen con evidentes fines de lucro, mediante el otorgamiento de una licencia que validaba las políticas, los mecanismos y las prácticas de la institución. Este sistema de licenciamiento otorgó licencia para lucrar a las instituciones que tenían estos fines. Las prácticas y modos de lucrar fueron validadas como mecanismos legítimos y ajustados a procesos aseguramiento de la calidad.

La regulación por la vía de la verificación y promoción de la calidad derivó en la Ley $\mathrm{N}^{\circ} 20.129^{2}$. Tal y como se ha verificado empíricamente por IPSOS

2 Ley $N^{\circ}$ 20.129: Ley de Aseguramiento de la Calidad de la Educación Superior, promulgada por la Presidencia de la República, en octubre de 2006. 
(2010) y como lo establece Brunner [6], no se debe desconocer que los procesos de acreditación han impactado de manera significativa en la gestión de las instituciones, en su preocupación y en la magnitud y profundidad de los esfuerzos por los temas de dirección y gestión estratégica, calidad, pertinencia y flexibilidad curricular, así como resultados de los procesos de enseñanza y aprendizaje. Del mismo modo, ha existido un énfasis en el perfeccionamiento y la evaluación de sus recursos humanos y en la administración de los recursos financieros. Sin embargo, el sistema nacional de aseguramiento de la calidad ha perdido credibilidad para garantizar la fe pública de la calidad de las instituciones y programas de pregrado al transformar la acreditación en un "commodity". Particularmente, la acreditación de instituciones con sedes de precaria calidad académica; la acreditación del 95\% de las carreras de pedagogías, algunas con evidentes carencias en sus sedes regionales, y la acreditación de algunas carreras de medicina con deficientes resultados en el examen médico nacional han configurado un panorama de desconfianza creciente en el sistema.

Por lo demás, dado que los procesos de acreditación se basan en los propósitos institucionales y en criterios de consistencia interna, la acreditación tampoco ha contribuido a generar una cultura de excelencia académica, sino que más bien a la búsqueda de la suficiencia o al logro de estándares mínimos para aprobar los requerimientos de los procesos de evaluación externa.

De este modo, tanto el licenciamiento como la acreditación, al adaptar sus mediciones a las características de las instituciones, han contribuido a validar el modelo de mercado. En efecto, con la argumentación de no generar clones de las instituciones de mayor tamaño y complejidad, no se han exigido estándares objetivos y cuantitativos. En virtud de lo anterior, ha existido un respeto casi dogmático por cualquier modelo de institución de educación superior, incluyendo la de universidades dedicadas a la venta de servicios docentes y títulos universitarios, cuyos propósitos evidentes han sido favorecer la obtención directa o indirecta de lucro para sus propietarios.

\section{Financiamiento de la educación superior}

Cabe consignar, en segundo lugar, que la consolidación del sistema nacional de educación superior ha tenido como estrategia fundamental el diseño de un sistema de financiamiento cada vez más orientado a la demanda. Mientras en 1981 el sistema y las instituciones se financiaban básicamente a partir del Aporte Fiscal Directo, en la actualidad la diversificación de ingresos es una realidad. Los aranceles por matrícula de pregrado, postgrado, y la venta de servicios conforman la estructura de ingresos a la cual se suman proyectos de inversión conseguidos en concursos del Ministerio de Educación y fondos para investigación.

Efectivamente, los recursos públicos asignados a ayudas estudiantiles se incrementaron durante el período 1990-2010 en más de 18 veces. De este modo, la matrícula ha llegado a una cobertura superior al 50\% de la población entre 18 y 24 años. Por lo demás, como lo indica Brunner [6], las personas con educación superior en el grupo de edad entre 25 y 34 años llegan a un 34\% en la actualidad. De esta manera el negocio de la educación superior bordea los USD 6.000 millones anuales, incluyendo los proveedores y empresas relacionadas al sistema. Como contraparte, los recursos destinados al Aporte Fiscal Directo e Indirecto han caído, en términos reales, sobre $\$ 200$ mil millones al año [20]. Este hecho deja de manifiesto que las políticas post gobierno militar simplemente afianzaron la lógica de mercado, con la excepción del programa Mejoramiento de la Calidad y Equidad de la Educación Superior (MECESUP), que ha sido el principal y, prácticamente, único respiro para sustentar inversiones en las instituciones del Consejo de Rectores de Universidades Chilenas.

Una mención especial merece la instauración del crédito con aval del Estado. Iniciativa que emergió como una solución a la creciente demanda por crédito solidario entre las universidades del Consejo de Rectores de Universidades Chilenas, y como una solución, además, para enfrentar la demanda de las instituciones privadas que solicitaban que sus estudiantes contaran con financiamiento crediticio. Su diseño consideró varios aspectos de difícil implementación: primero, la definición de un arancel de referencia a partir de una fórmula diseñada "ex profeso" para que las instituciones de mayor prestigio quedaran clasificadas con los aranceles más altos. De igual modo, el arancel de referencia ha sido una pesada carga para la totalidad de las instituciones del Consejo de Rectores que cuentan con 
altos estándares de calidad y cantidad en su cuerpo académico, por lo que los aranceles de referencia no representan los aranceles reales; segundo, se dejó al funcionamiento del mercado financiero la definición de la tasa de interés pensándose, probablemente, en una tasa razonable para un crédito básicamente sin riesgo, pero las tasas alcanzadas estuvieron significativamente sobre lo esperado, llegándose a niveles de una tasa real del $6 \%$.

Dicha tasa genera varias paradojas. Primero, para una familia resulta más conveniente vender su casa o bien raíz a fin de disponer del dinero que le permita pagar la totalidad de la carrera, y endeudarse en un crédito hipotecario para luego recuperar una casa o bien raíz equivalente. En su versión simplificada, esta opción de arbitraje financiero puede proporcionar, con las tasas vigentes en el mercado financiero, un ahorro neto del $2 \%$ anual. Segundo, dos hijos de una misma familia, uno con crédito solidario y el otro con crédito con aval del Estado, que estudien la misma carrera, tendrán que asumir una diferencia en tasa de interés real equivalente al $4 \%$ anual. Tercero, la rentabilidad esperada de la educación superior para un país desarrollado bordea el 8,5\% [5] con lo cual una tasa del $6 \%$ se apropia de la mayor proporción de la rentabilidad privada esperada de largo plazo de seguir estudios superiores.

El crédito con aval del Estado ha sido la máxima apuesta para desarrollar el mercado de la educación superior y su diseño e implementación se gestó 16 años después de terminar el gobierno militar. No sólo se trató de desarrollar el mercado del pregrado, sino que también el mercado financiero. Aunque previamente varias instituciones privadas habían otorgado, por años, créditos directos a sus estudiantes sin fiscalización de ningún tipo.

En tercer lugar, las tareas de investigación, desarrollo e innovación se han solventado mediante la lógica de concursos. De esta manera se ha impulsado el establecimiento de centros de excelencia, prácticamente, en cada región del país, programas asociativos de investigación, programas de formación de postgrado en Chile y fuera de Chile, junto a los tradicionales y escasos fondos anuales destinados a la investigación científica y tecnológica e investigación aplicada, vía los Fondos de la Comisión Nacional de Investigación Científica y Tecnológica (CONICYT).
Los montos dirigidos a estos fines eran hasta hace unos pocos años, el equivalente sólo a un tercio del promedio de los países de la Organización para la Cooperación y el Desarrollo Económicos (OCDE). Ciertamente, con los nuevos fondos para la innovación y competitividad regional los recursos se han incrementado significativamente. Sin embargo, la investigación y el desarrollo requieren de capacidades instaladas tanto en términos de recursos humanos altamente calificados, como también de infraestructura y equipamiento, cuestiones que en un marco de exiguos aportes basales para sustentar programas de largo plazo sólo permiten niveles modestos de progreso. Por lo mismo, Chile sigue siendo un exportador básicamente de materias primas, lo que representa quizás en su expresión más profunda y concreta el nivel del desarrollo científico y tecnológico del país, el que es claramente deficiente para insertarse competitivamente en la sociedad del conocimiento.

El énfasis estratégico dado al sistema chileno de educación superior ha derivado en aranceles de matrícula que, en términos relativos, están entre los más elevados del mundo. Por ejemplo, el arancel promedio de las universidades chilenas corresponde al $41 \%$ del ingreso per cápita del país, mientras que en los Estados Unidos esa proporción es del 28\%, en Australia es el 12\% y en Canadá es el 10\% [19].

Junto a la definición de aranceles, varias instituciones han desarrollado su propio mercado financiero con sus estudiantes. Es decir, han aprovechado la oportunidad de disponer de un "mercado cautivo". Los términos de los créditos otorgados a los alumnos prácticamente no han tenido ningún tipo de control ni evaluación en términos de: tasas de interés, condiciones de la reprogramación, plazos otorgados, etc. Asimismo, la relación entre universidades y sus empresas relacionadas ha carecido de control de los precios de transferencia, o la desviación de lucro hacia esas entidades. En consecuencia, el sistema ha operado en ausencia de la regulación más elemental.

La búsqueda de lucro en el sistema, así como los altos niveles de rentabilidad han quedado en evidencia con las compras de universidades realizadas por grupos económicos y de inversión tanto nacionales e internacionales. 


\begin{abstract}
Análisis del modelo de educación superior chileno Complementariamente, es importante evaluar si los objetivos estratégicos pregonados públicamente por los ideólogos del modelo vigente en Chile se cumplen o no. Por lo tanto, la cuestión a dilucidar es: si efectivamente existe o no libertad para elegir; si el modelo genera bienes valiosos para los agentes; y si, finalmente, existen niveles satisfactorios de cobertura, calidad y equidad en el sistema chileno de educación superior.
\end{abstract}

Para este efecto, se realizarán una serie de preguntas estratégicas a las que se intentará dar respuesta, considerando los determinantes estructurales, los efectos y los alcances de los elementos que subyacen en dichas respuestas.

La primera pregunta es: ¿los estudiantes de la educación superior chilena tienen la libertad para elegir realmente en dónde realizar sus estudios terciarios? Esta interrogante alude al corazón del sistema chileno, ya que la libertad para elegir implica dotar a cada potencial estudiante de la capacidad y del poder para obrar según su propia voluntad, haciéndose responsable de su elección.

La verdad sea dicha, y es que en la educación superior chilena no hay libertad de elección salvo para los miembros de los grupos altamente privilegiados desde la perspectiva social y económica.

En efecto, la educación recibida en enseñanza básica y media es el principal determinante de los resultados de los procesos de selección universitaria. Además, considérese que la propia calidad de la formación recibida en enseñanza básica y media está determinada por la condición social y económica de las personas.

Luego, con las excepciones normales en el campo de las ciencias sociales, la condición socioeconómica determina de manera significativa la calidad de la enseñanza básica y media, la que a su vez condiciona los resultados de los procesos de selección universitaria. Por ejemplo, los colegios Top-60 en la prueba de selección universitaria tienen promedios en dicho test que permiten a sus egresados elegir, de verdad, qué y dónde estudiar. $\mathrm{Al}$ respecto, en la prueba rendida el año 2010, el 95\% de estos colegios eran particulares pagados; el $3,4 \%$ fueron colegios municipalizados; y el 1,6\% de los casos, equivalente a un colegio era particular subvencionado [8]. Lo anterior ilustra que la relación entre nivel socioeconómico y resultados en la prueba de selección es clara y evidente, por lo cual las condiciones de origen de los estudiantes pueden ser más determinantes que sus talentos para ingresar y elegir una institución de educación superior.

Ahora bien, la misma situación social y económica pone una traba adicional al mérito académico. En efecto, aunque los estudiantes logren derrotar sus condiciones de entrada u origen social y económico, de igual modo deben disponer de los recursos adicionales a los costos de matrícula para garantizar su manutención personal durante los años que duren sus estudios universitarios, cuestión que restringe sus opciones reales de elección.

Por lo tanto, no cabe duda que las posibilidades de acceso y la calidad de la educación disponible para los jóvenes de Chile se asocian indisolublemente a la condición social y económica de su núcleo familiar.

Por cierto, dos jóvenes de igual talento académico y calidad intelectual no tienen las mismas oportunidades de elección si sus condiciones económicas son significativamente distintas. El joven con peor condición social y económica tendrá, con las excepciones que confirman la regla, una educación de menor calidad en la enseñanza básica, media y universitaria. Consecuentemente, recibirá menores ingresos que los jóvenes de igual talento, pero de una condición social y económica significativamente superior. Habrá movilidad social, ya que el joven de menores recursos "saldrá adelante en la vida", pero sus oportunidades no se acercarán siquiera en lo básico a las opciones de otras personas de igual talento, cuya diferencia fundamental son las condiciones de nacimiento. En efecto, Benavente, Rappoport y Meller [2] muestran que en un conjunto de carreras universitarias hay diferencias de remuneraciones significativas dependiendo de la universidad en la cual se obtuvo el título correspondiente.

En consecuencia, a igual talento una peor condición socioeconómica implica estudiar en un colegio con peores resultados académicos, lo que, a su vez, conlleva a acceder a una educación terciaria de menor selectividad y, consecuentemente, a un trabajo peor remunerado. 
La situación es tanto o más grave cuando se incorpora al análisis el componente regional. Un estudiante talentoso de los quintiles más desposeídos de Arica o Punta Arenas no tiene las mismas oportunidades de elección que un estudiante del mismo talento y de las mismas condiciones que vive en la ciudad de Santiago. Por ejemplo, entre los colegios Top-60 de la última prueba de selección universitaria no existe ni siquiera un colegio de ninguna de las regiones de Chile que sea municipalizado o particular subvencionado [8].

Luego, el modelo actual no cumple con su objetivo primario de dar a las personas libertad para elegir. En efecto, en este sistema de educación superior las personas eligen lo que pueden y lo que pueden está condicionado, antes que nada, por la cuna en la cual nacieron.

La reflexión anterior parece invalidar, en sí misma, la pertinencia estratégica del modelo actual. En cualquier caso, es importante avanzar en la segunda interrogante asociada a los objetivos del sistema de educación superior: ¿el modelo genera bienes valiosos para los individuos y para la sociedad? Esta pregunta alude a otra cuestión esencial en tanto la existencia de bienes privados, es decir, bienes valiosos para los individuos se puede asociar a una lógica de coordinación mediante el mercado y el mecanismo de precios; sin embargo, la potencial existencia de bienes públicos generados por el modelo rompe con la pertinencia de una lógica estricta de mercado y da lugar a la necesaria existencia de un rol determinante y activo del Estado.

En la sociedad del conocimiento tanto capital como trabajo son requeridos para la producción de bienes, pero el conocimiento es la fuente de la ventaja competitiva en la nueva economía. Por lo tanto, desde esta perspectiva, la educación superior debe asumir un conjunto de desafíos centrales. Primero, las instituciones de educación terciaria deben constituirse en un elemento básico para generar mayores niveles de competitividad en el país. En efecto, en la sociedad del conocimiento y en un ambiente globalizado la formación de capital humano avanzado, así como la investigación, el desarrollo y la innovación constituyen pilares fundamentales de la ventaja competitiva para la nación, las organizaciones y las personas. Segundo, las instituciones de educación superior deben constituirse en una fuente esencial de oportunidades de formación continua y movilidad social. Ciertamente, la rentabilidad privada de la educación superior es aún significativa, particularmente en los países emergentes. Tercero, las instituciones de educación superior deben velar por la calidad y pertinencia de su oferta académica, cuestión de la mayor importancia para generar programas de formación e investigación que, efectivamente, impacten sobre la capacidad competitiva del país y permitan la inserción exitosa de jóvenes de familias vulnerables en el mercado del trabajo. Cuarto, las instituciones de educación superior deben vincularse con su medio aportando al desarrollo de la cultura, las artes, las letras y el desarrollo territorial [23].

Es indudable que la educación superior chilena puede generar bienes privados, pero también genera bienes públicos. Por ejemplo, un año más de escolaridad tiene un impacto sobre la productividad individual que bordea entre 6 y $15 \%[9,1,18]$, cuestión que debería conducir a una mejor remuneración para los individuos, proporcionando a éstos una rentabilidad privada; pero ese mismo año adicional de escolaridad implica un incremento del potencial del producto interno bruto en proporciones que, en el largo plazo, resultan altamente significativas, contribuyendo de esta forma a un mayor bienestar y progreso para la sociedad en su conjunto.

Por su parte, la creación de conocimiento asociada a investigación, desarrollo e innovación permite la generación de nuevos productos y tecnologías $\mathrm{y}$, por ende, tiene una alta rentabilidad privada y social. Por ejemplo, los trabajos de Nadiri [21] y de Lichtenberg y Siegel [16] muestran rentabilidades privadas superiores al $20 \%$ para las inversiones en investigación y desarrollo en diferentes sectores económicos. Por su parte, los trabajos de Frantzen [12] y de Lichtenberg y Van Pottelsberghe [17] muestran una rentabilidad social superior al 50\% para las inversiones en investigación y desarrollo. Esto no sólo ocurre en los países desarrollados, ya que Benavente [3] demuestra que la situación se repite en una economía emergente como la de Chile. Por cierto, la investigación, el desarrollo y la innovación son bienes que proporcionan beneficios privados, pero son también bienes públicos, en tanto generan beneficios para toda la sociedad. 
La educación terciaria constituye una fuente de movilidad social con una rentabilidad privada que es aún atractiva para las personas, principalmente en los países emergentes $[10,11]$, incluso en localidades o regiones distantes del centro del país [7]. Por lo demás, la formación de capital humano avanzado permite la consolidación territorial de las distintas regiones de un país. Como lo plantean Bloom, Canning y Chan [4], la educación superior tiene una serie de beneficios para una sociedad tanto desde la perspectiva económica como desde la perspectiva social. Los beneficios económicos se asocian a: mayor recaudación de impuestos; mayor potencial de productividad para el país; mayores niveles de demanda agregada; mayores niveles de ahorro; mayor flexibilidad de la fuerza de trabajo; entre otros similares. Por su parte, los beneficios sociales se asocian a: menores tasas de criminalidad; mejor calidad de vida cívica; mayor cohesión social; aprecio por la diversidad; mejores condiciones de salud y expectativas de vida; entre otros beneficios similares.

En este punto de la reflexión emergen, con fuerza, tres consideraciones básicas. Primero, en Chile existe la tendencia a considerar a la educación superior sólo como docencia, despreciando la relevancia y el rol esencial que las instituciones terciarias tienen en la investigación, el desarrollo y la innovación. Asimismo, no se suele dimensionar el verdadero valor del rol de la vinculación con el medio de las instituciones de educación superior. Probablemente, el hecho de que muy pocas instituciones chilenas cumplan estos roles con solvencia hace que se tienda a menoscabar conceptualmente y en los hechos concretos los roles de formación de postgrado, investigación y vinculación con el medio. Segundo, la educación superior es un bien cuasipúblico, que tiene componentes de bien privado y este hecho no puede ni debe ser negado; pero tampoco puede ni debe ser negado su impacto como bien público, ya que incluso desde la perspectiva más reduccionista de la docencia de pregrado y contemplando sólo los impactos económicos se pueden comprender los efectos sobre la productividad del país, la recaudación fiscal, y los mayores niveles de ahorro que genera la educación terciaria. Ahora bien, la educación superior tiene un rol vital como bien público, mucho mayor todavía cuando se incorpora la visión más integradora de los roles de investigación, desarrollo e innovación. Tercero, el conocimiento tiene valor en sí mismo, porque en el conocimiento está la fuente del progreso no sólo económico, sino que las bases de la cultura, y del desarrollo de la sociedad. El afán y la vocación por aprender e investigar son el sustento del desarrollo de la humanidad; ciertamente, muchos de los principales descubrimientos, inventos y reflexiones no se llevaron a cabo bajo la lógica de la búsqueda de la productividad, sino que emergieron del talento, ingenio y la curiosidad de personas con mentes entrenadas para crear y proyectar su saber.

En consecuencia, el modelo chileno actual de educación superior es reduccionista, pues minimiza los roles fundamentales de las instituciones universitarias en la sociedad del conocimiento y desconoce, convenientemente para los grupos de interés representados en el "establishment", la naturaleza esencialmente pública de los bienes generados por la educación terciaria.

La tercera pregunta desde la mirada estratégica es: ¿existen niveles satisfactorios de cobertura, calidad y equidad en el modelo chileno de educación superior? Esta pregunta se vincula con una revisión más profunda y crítica, de los resultados más difundidos del modelo.

Respecto de la cobertura, se sabe que ésta alcanza a más de un millón de estudiantes, lo que representa más del $50 \%$ de la población entre 18 y 24 años. En efecto, en 20 años se quintuplicó la cobertura en el $30 \%$ de las personas con menores ingresos; lo cual es sin duda una buena noticia. Sin embargo, sólo un $19,1 \%$ de los estudiantes del $10 \%$ más vulnerable de la población ingresa a la educación superior; en tanto que un 93,3\% del decil más rico logra ingresar a la educación terciaria [15]. Los más desposeídos no sólo ingresan en menor proporción a la educación superior, sino que la posibilidad de ingresar a las universidades, en lugar de a institutos profesionales o centros de formación técnica, es mayor para las personas de mejor situación socioeconómica.

Junto a esta cobertura que muestra progreso y, a la vez, falta de equidad, es menester considerar que la deserción en la educación superior chilena es del $52 \%$ [25]. Este hecho implica que más de la mitad de los estudiantes que ingresan a estudiar educación terciaria nunca se gradúan. La duración real de las carreras suele ser 1,5 veces la duración teórica o ideal. 
Sin duda, estas cifras dejan de manifiesto que el sistema fracasa en su cometido, ya que si bien tiene las puertas abiertas para el ingreso, las puertas de la salida al éxito están llenas de obstáculos. Por eso, cuando se dice que siete de cada diez personas que ingresan a la educación superior son primera generación familiar en lograrlo, resulta fundamental conocer la respuesta a: ¿cuántas de esas siete personas finalmente se gradúan y en cuánto tiempo lo logran?

Probablemente, para algunas personas, sea mejor no ingresar a la educación superior, que salir de ésta sin éxito, con los sueños y las ilusiones rotos, y con un endeudamiento que acechará y limitará su consolidación familiar y económica.

El sistema chileno muestra una cobertura creciente y en progreso, pero ésta es una falsa cobertura. En efecto, más de la mitad de las personas que están en educación superior no se graduarán nunca. En cuanto a equidad el sistema es claramente deficiente.

Respecto de la calidad, todas las universidades privadas pasaron por largos procesos de evaluación por parte del Consejo Superior de Educación. No obstante, este hecho no ha sido garantía de calidad. Por cierto, instituciones licenciadas no fueron capaces de pasar posteriores procesos de acreditación, dejando en evidencia que el licenciamiento fue prematuro, muy flexible, o simplemente las instituciones cambiaron radicalmente sus proyectos educacionales una vez que lograron su autonomía. La misma acreditación que en algún momento mostró méritos innegables en cuanto a su impacto en los procesos de mejoramiento en las instituciones [14], cada vez ha perdido mayor vigor y credibilidad.

Por ejemplo, en los procesos de acreditación institucional de fines de 2010, todas las instituciones que postularon fueron acreditadas. Lo anterior podría implicar que ha existido un mejoramiento notable en las instituciones de educación terciaria en Chile o que, simplemente, la vara de medida y los niveles de exigencia han caído a niveles lamentables. Ahora bien, cuando se observa el estado y el bajo nivel de calidad con el cual operan sedes de instituciones llamadas universitarias, sobre todo en las regiones del país, queda en evidencia que el sistema de aseguramiento de la calidad en Chile no está garantizando el cumplimiento de estándares mínimos, al menos en las sedes regionales.
Sin embargo, esto no ocurre sólo en las acreditaciones institucionales. Recientemente, a fines del año 2010, una institución universitaria acreditó su carrera de Medicina ante una agencia privada, por dos años. Lo que debería significar que en dicha carrera hay garantía de calidad. Sin embargo, el $48 \%$ de sus titulados, luego de siete años de estudio, reprobó el examen médico nacional, dejando en evidencia su escaso nivel de preparación y conocimiento [24]. Una reflexión adicional a este marco de referencia y análisis es saber ¿a quiénes atenderán estos médicos, tan deficientemente formados? Probablemente a quienes no puedan costear los servicios de médicos de mayor preparación, jerarquía académica e intelectual.

La relación cliente-proveedor entre instituciones de educación superior y agencias privadas, de acreditación de carreras y programas, genera una relación intrínsecamente compleja, con la existencia de riesgos implícitos similares a los que la evidencia empírica muestra en el caso de las instituciones privadas clasificadoras de riesgo, cuya responsabilidad en la reciente crisis económica mundial ha sido significativa (Instituto Iberoamericano de Mercado de Valores [13]). Efectivamente, si quien evalúa es una institución con fines de lucro, cuyos beneficios principales se obtienen de la venta de servicios a quienes son los evaluados y si, además, la relación de evaluación es de largo plazo y puede ser una relación de clienteproveedor permanente, entonces es posible que exista riesgo moral, ya que las consecuencias de evaluaciones poco rigurosas no serían plenamente asumidas por las agencias de acreditación. Por cierto, en un marco de asimetría de información, sólo en el largo plazo, el mercado y la propia Comisión Nacional de Acreditación, podrían descubrir conductas no apropiadas a los fines del sistema nacional de aseguramiento de la calidad.

\section{CONCLUSIONES}

El sistema de educación superior chileno muestra una cobertura creciente, pero esconde la realidad de altos niveles de deserción y una prolongada permanencia o duración excesiva de los estudios. Por lo demás, no hay equidad en el acceso, y la calidad institucional de las carreras y de los programas no está garantizada en el contexto de un sistema de aseguramiento de la calidad que pierde consistencia y credibilidad. 
Con todas estas reflexiones sería fácil demonizar al mercado como el responsable de los males de la educación superior en Chile, pero el culpable no es el mercado.

Al mercado no se le puede pedir imposibles. Efectivamente, si como se ha dicho en las ideas anteriormente expuestas, la condición socioeconómica determina las posibilidades de elección real de los estudiantes; si los costos de la educación no se pueden estandarizar ya que el capital social, económico y cultural de la población estudiantil es un determinante estructural de sus posibilidades de aprendizaje; si no existe una perfecta movilidad de recursos en el sistema de educación superior; si existen costos elevados de traslado para los usuarios del sistema; y si la asimetría de información que existe en el sistema no permite a los estudiantes y a sus familias adoptar, necesariamente, las mejores decisiones, entonces no se le puede pedir al mercado que obre no sólo uno, sino que varios milagros simultáneos.

\section{AGRADECIMIENTOS}

El autor agradece el aporte otorgado a través del proyecto FONDECYT 1090116, financiado por la Comisión Nacional de Investigación Científica y Tecnológica de Chile.

\section{REFERENCIAS}

[1] A. Bassanini and S. Scarpetta. "Does human capital matter for growth in OECD countries? A pooled mean-group approach". Economics Letters. Vol. 74, pp. 399-405. 2002.

[2] J. Benavente, D. Rappoport y P. Meller. "Rankings de universidades chilenas según los ingresos de sus titulados". Banco Central de Chile. Documentos de Trabajo. № 306. Diciembre 2004.

[3] J. Benavente. "The Role of research and innovation in promoting productivity in Chile". Economics of Innovation and New Technology. Vol. 15, Issue 4-5, pp. 301-315. 2006.

[4] D. Bloom, D. Canning and K. Chan. "Higher education and economic development in Africa”. Harvard University. 2006.

[5] R. Boarini and H. Strauss. "The Private Internal Rates of Return to Tertiary Education: New Estimates for 21 OECD Countries". OECD
Economics Department Working Papers. Vol. 591. OECD. Economics Department. 2007.

[6] J.J. Brunner. "Taller Aequalis". 2011. URL: http://www.aequalis.cl/wp-content/ uploads/2011/08/Presentación-José-JoaquínBrunner.pdf.

[7] J.J. Brunner, J. Bonnefoy, G. Elacqua y S. González. "Capital Humano en la Región de Tarapacá". Universidad Adolfo Ibáñez, p. 75.2006.

[8] DEMRE 2011. "Proceso de admisión 2011, resultados de selección”. Universidad de Chile. Vicerrectoría de Asuntos Académicos. Departamento de Evaluación, Medición y Registro Educacional. Santiago, Chile. 12 de enero de 2011.

[9] S. Dowrick. "Ideas and education: Level or Growth Effects?" Thirteenth Annual East Asian Seminar on Economics Productivity, Melbourne, June 20-22, 2002.

[10] S. Duryea and C. Pagés. "Human Capital Policies: What they Can and cannot do for Productivity and Poverty-reduction in Latin America". Forthcoming in: American Foreign Economic Relations: Policy Dilemmas and Opportunities. North South Press. Miami, United States. 2001.

[11] N. Fleet. "Rentabilidad privada de la educación superior". Working Paper. Comisión Nacional de Acreditación. Vol. 5, p. 29. Santiago, Chile. 2007.

[12] D. Frantzen. "Innovation, international technological diffusion and changing influence of R\&D on productivity". Cambridge Journal of Economics. Vol. 24, pp. 193-210. 2000.

[13] Instituto Iberoamericano de Mercado de Valores. "Estudio sobre la transparencia de los emisores en Iberoamérica". 2010. URL: http://www.iimv.org/estudios.htm

[14] IPSOS 2010. "Informe final: Estudio exploratorio sobre efectos de la acreditación institucional en la calidad de la educación superior en Chile". Preparado para la Comisión Nacional de Acreditación, Santiago, Chile. Abril 2010.

[15] J. Lavín. "El modelo de educación superior para el Chile del futuro". Presentación del Ministro de Educación en el salón de honor del Congreso Nacional. 13 de enero de 2011.

[16] F. Lichtenberg and D. Siegel. "The impact of R\&D investment on productivity- New 
evidenve using R\&D-LRD Data”. Economic Inquiry. Vol. 29, pp. 203-228. 1991.

[17] F. Lichtenberg and B. Van Pottelsberghe. "International R\&D Spillovers: A Re-Examination”. NBER Working paper. N ${ }^{\circ} 5668$. Issued in July 1996.

[18] N. Mankiw, D. Romer and D. Weil. "Contribution to the empirics of economic growth". Quarterly Journal of Economics. Vol. 107, Issue 2, pp. 407-437. 1992.

[19] P. Meller. "El costo y financiamiento de la educación superior en Chile". Columna El Mercurio. 2011. URL: www.cieplan.org/ noticias/detalles.tpl?id $=152$

[20] P. Méndez. "Sistema de financiamiento de la educación superior chilena". Claustro Triestamental Universidad de Tarapacá. 2011.

[21] M. Nadiri. "Innovations and Technological Spillovers". NBER Working Paper. $\mathrm{N}^{\circ}$ W4423. 1993.
[22] OCDE. "Revisión de políticas nacionales de educación. La educación superior en Chile". OCDE y Banco Mundial. 2009.

[23] E. Rodríguez-Ponce. "Las universidades en la sociedad del conocimiento", pp: 35-68. En A. Arata Andreani y E. Rodríguez Ponce, editores. Desafíos y perspectivas de la dirección estratégica de las instituciones universitarias. Ediciones CNA Chile, p. 549. 2009.

[24] E. Rodríguez-Ponce. "Sistema de acreditación y calidad de la educación superior". Presentación efectuada en Sala de Lectura, de la Cámara de Diputados, Congreso Nacional. Santiago, Chile. 23 de mayo de 2011.

[25] J. Ugarte. "Desafíos de la educación superior chilena". 2011. URL: http://www.cedus.cl/?q $=$ node $/ 1634$ 